\title{
How Can We Investigate What We Cannot Scale? Introducing the Concept of Synoptic Models for Fluvial Processes
}

\author{
Ivo BASELT \\ Universität der Bundeswehr München, Hydromechanics and Hydraulic Engineering, \\ Neubiberg, Germany \\ $\triangle$ ivo.baselt@unibw.de
}

\begin{abstract}
To investigate fluvial processes under laboratory conditions the application of similarity laws is a well-accepted experimental method. Studying natural systems in scaled physical models is usually limited by the laboratory's infrastructure and by similarity validities, which confines the model's realisable geometric dimension. Using similarity laws becomes barely feasible if complex geomorphic fluvial events are studied with physical laboratory models, especially due to the steep slope and broad grain size distribution. Nevertheless, computational models predicting the outcomes of these hazard events require reliable data sets for calibration and validation. In this work, we present a synoptic model approach to overcome this dilemma. Derived from other research disciplines, we will discuss, how the synoptic functionality could be applied to fluvial problems based on the example of the 2017 Piz CengaloBondo landslide.
\end{abstract}

Keywords: similarity laws, geomorphic fluvial systems, physical laboratory models, analogue models, synoptic model approach.

\section{EXTENDED ABSTRACT}

Using similarity laws to investigate research questions in the field of hydromechanics and morphodynamic is a well-known experimental method. By transferring the properties of the investigated prototype to a downsized physical laboratory model the associated workflow usually begins by identifying an appropriate length scale. In general, the design of physical laboratory models is limited by several conditions. The model's geometric dimensions cannot be too large 
because the lab infrastructure (space, discharge) is restricted. Other limits are the model's cost, its manageability, or safety at work. On the contrary, the model's dimension can also not be too small without violating specific similarity validities (state of flow, surface tension, transcendence of cohesive grain size, similarity of sediment transport). Normally, a possible range of length scale factors can be determined that allows the realisation of models under laboratory conditions, which becomes much more difficult when geomorphic fluvial events should be studied with laboratory models. Landslides, torrential flows, and debris flows normally occur in areas with large topographic inclinations. The total elevation difference from flow initiation until deposition is often from a similar magnitude than the horizontal runout distance in these fluvial events. An appropriate realisation of the real system in a scaled model often fails due to the available vertical space. Furthermore, downscaling the flow depth and the grains size induce insurmountable problems of surface tension and cohesive effects because gravitational multiphase mass flows often contain a noticeable amount of fine sediments, which cannot be scaled properly. Moreover, despite requiring only a few dimensionless numbers for scaling river systems (Fr, Re, We), additional dimensional numbers like Savage, Bagnold, or Friction must be considered in gravitational mass flows, which impedes the realisation.

Nevertheless, there is an essential need to study complex geomorphic fluvial processes. Computational models are used to create hazard maps, adjusting prewarning systems, and implementing effective countermeasures to reduce the risk of such hazard events. Detailed data sets are therefore essentially required for calibration and validation. Unfortunately, field data is rare and former laboratory experiments were mainly conducted in straight flumes under very idealized conditions. To overcome this dilemma, novel experimental methods for complex geomorphic fluvial processes are needed to create data sets for calibration and validation of holistic computational models. Already applied in meteorology, product design, or electric mobility systems synoptic model approaches help to find isomorph conclusions by studying relevant attributes in one or more interrelated analogue systems (replacement systems) instead of upscaling measured physical variables from a model to the prototype. This synoptic approach could include physical models, computational models, analytical solutions, empirical approaches or laboratory and field measurements. The finding from all interrelated replacement systems must be finally merged in a conclusion of the studied problem.

In this work, we present some first conceptual ideas on how to transfer the concept of synoptic models to a potential application for fluvial processes. After we give an overview of the techniques and functionality of synoptic models, the concept is explained exemplary based on the 2017 Piz Cengalo-Bondo landslide cascade. Since the total original system cannot be scaled to a physical laboratory model, we identify relevant attributes in the original system which might serve as analogue sources. This could be the type of failure (rockfall), the overall terrain characteristic and sectionwise slopes, areas for erosion and deposition, and mobility. Then, we propose to split the total system for the Bondo landslide cascade into four analogue subsystems to investigate the relevant attributes in interrelated replacement systems. Because the initial rockfall and the following fragmentation follow stochastic behaviour, this process might be investigated with data from field surveys or with empirical approaches. How the fragments entrain the glacier's ice and how the ice is liquefied due to shearing could be studied in laboratory rheological tests. An existing computational multi-phase mass flow model should be used to study the flow properties of the evolved channelized debris flow in the narrow valley. Finally, a sophisticated scaled laboratory model could represent the last part of the Bondo landslide and enable studying the deposition morphology in the city of Bondo. Following the principles of synoptic modelling, specific model parameters can be set unleashed in the interrelated replacement systems, like length scale, particle size, or bathymetry. The combined results from all analogue models create a suitable data set serving for calibration and validation of holistic simu- 
lations. Even if a real application is still pending, this work provides some first novel ideas on how to create calibration and validation test cases for computational models by deducing isomorph conclusions from a synoptic model approach.

Received 22 March 2021

Accepted 12 April 2021 